\title{
Synthesis of New Substituted Purines and $[1,2,4]$ Triazolo- [3,4-i]purine Derivatives
}

\author{
W.M. Basyouni", K.A.M. El-Bayouki and W.M.A.Tohamy \\ National Research Centre, Cairo, Egypt.
}
7 REATMENT of the 6-hydrazinopurine derivative 2a with is othiocyanates followed by chloroacetyl chloride afforded, the1,3-thiazolidine-2,4-dione-2-[9-aryl-9H-purin-6-yl] hydrazones (5). Moreover, the 2-substituted [1,2,4]triazolo[3,4-i]purines 9 and 10 were synthesized by reacting $2 \mathrm{a}$ with aldoses and cyclizing the obtained product.

Keywords: Hydrazinopurine, 1,3-Thiazolidine-2,4-dione and Triazolo $[3,4-\mathrm{i}]$ purines.

Purines have successfully been employed in medicine for some decades. Whereas, many purine derivatives have been reported to have a wide range of biological activities.

Thus, the purine nucleus is found in a wide variety of biologically active molecules including nucleotides, enzyme cofactors, intracellular second messengers and pharmaceutical agents ${ }^{(1)}$.

Due to the fundamental role of purines in nucleic acid chemistry and cellular biochemistry, the possibility of using several purine derivatives as chemotherapeutic agents in the treatment of malignant diseases has been extensively investigated. Modified purines bearing substituents at the 2-, 6and/or 9-positions have been associated with a wide variety of interesting biological properties. For instance, they find application as cyclin-dependent kinase inhibitors ${ }^{(2-6)}$, adenosine receptor antagonists ${ }^{(7-9)}$, modulators of multidrug resistance $^{(10)}$ and as antiviral ${ }^{(11)}$ and antineoplastic agents ${ }^{(12)}$.

On the other hand, purines bearing $\mathrm{C}$-substituents containing functional groups $\left(\mathrm{OH}, \mathrm{NH}_{2}, \mathrm{COOH}\right.$..., etc.) would provide new exploitable moieties for interactions with complementary nucleobases, enzymes, or receptors while still preserving higher stability towards enzymatic degradation due to the presence of a C-C bond in position- $6^{(13)}$.

$C$-Nucleosides are important targets in organic synthesis due to their potential value as therapeutic agents and biochemical probes. A number of nucleoside analogues have been found to show a broad spectrum of biological activity and have stimulated considerable interest as potential antitumour and/or antiviral agents $^{(14)}$.

*Corresponding author, E-mail: w_basyouni2002@yahoo.com 
Also, numerous natural or synthetic nucleosides derived from a five membered-ring nucleobase and displaying a potent antitumoral or antiviral activity, e.g. thiazofurin, ribavirin and pyrazomycin B. have been described ${ }^{(15)}$. Heterocycles carrying carbon-carbon linked alditoyl chains constitute an important class of acyclic C-nucleosieds because of their various biological activities and medicinal applications ${ }^{(16)}$. Furthermore, several 2'- $C$-methyl purine nucleoside analogs with potent inhibitory activity against hepatitis $\mathrm{C}$ virus (HCV) NS5B polymerase have been synthesized and identified ${ }^{(17)}$. Fluorinated analogues of natural substances are mainly of interest in bioorganic chemistry, since single fluorine atom or trifluoromethyl groups introduced in place of hydrogen atoms often increase the biological activity of the parent compounds $^{(18)}$.

Therefore, the present work is intended for synthesizing newer purines and their corresponding [1,2,4]triazolo[3,4-i]purine derivatives for their expected useful biological properties.

\section{Results and Discussion}

When the 6-chloropurine derivative $(1)^{(19)}$ was allowed to react with hydrazine hydrate or methyl hydrazine, the corresponding: 1-(9-(4fluorophenyl)-9H-purin-6-yl) hydrazine (2a) and 1-(9- (4-fluorophenyl) -9Hpurin- 6-yl) -2- methyl-hydrazine (2b), respectively were smoothly obtained in good yields.
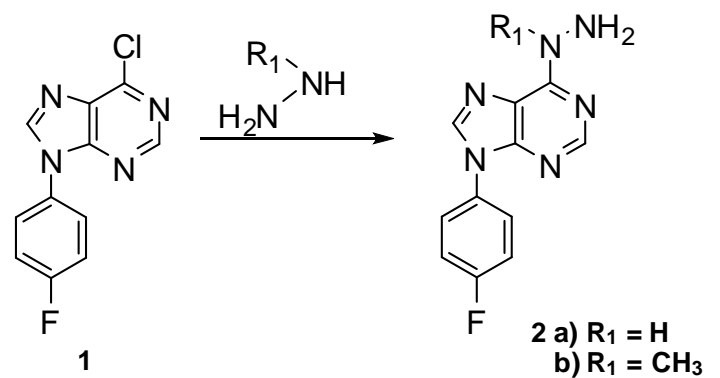

The present work was continued to probe the reactivity of the $\mathrm{NHNH}_{2}$ moiety in product (2a) towards some alkyl- and aryl-isothio cyantes. Thus, when the hydrazide (2a) was allowed to react with methyl-, ethyl-, propyl-, n-butyl-, cyclohexyl-, and phenyl isothiocyantes the corresponding 2-[9-(4-fluorophenyl)$9 \mathrm{H}$-purin-6-yl]- $\mathrm{N}$-substituted-hydra- zinecarbothioamide derivatives (3a-f) were obtained in good yields (Scheme 1).

Infrared absorption spectra of products (3) were characterized by the presence of $v_{\mathrm{NH}}$ and $v_{\mathrm{C}=\mathrm{S}}$ around 3260-3166 and 1228-1295 $\mathrm{cm}^{-1}$ regions, respectively. Also, their ${ }^{1} \mathrm{H}-\mathrm{NMR}$ spectra accorded well their proposed structures.

Egypt. J. Chem. 56, No. 1 (2013) 


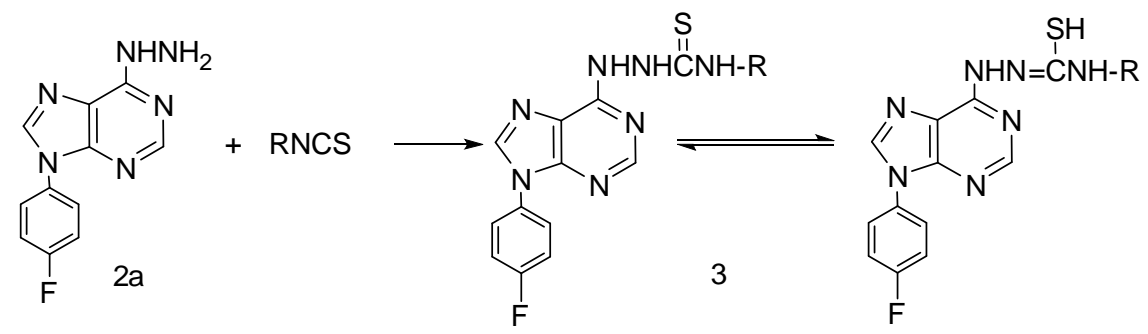

\begin{tabular}{lccc}
3 & $\mathrm{R}$ & 3 & $\mathrm{R}$ \\
\hline a) & $\mathrm{CH}_{3}$ & d) & $\mathrm{C}_{4} \mathrm{H}_{9}-\mathrm{n}$ \\
b) & $\mathrm{C}_{2} \mathrm{H}_{5}$ & e) & $\mathrm{C}_{6} \mathrm{H}_{11}$ \\
c) & $\mathrm{CH}_{2}-\mathrm{CH}=\mathrm{CH}_{2}$ & f) & $\mathrm{C}_{6} \mathrm{H}_{5}$
\end{tabular}

Scheme 1

When products (3) were allowed to react with ethyl 2-bromoacetate, the ethyl $\{[\{[9-(4-f l u o r o p h e n y l)-9 H-6-y l]$ hydrazono $\}$ (substitutedamino)- ethyl] sulfanyl $\}$ acetates $(4 \mathrm{a}-\mathrm{f})$ were obtained rather than the expected produts $2-\{[9-(4-$ fluoro-phenyl) $-9 H$ - purin-6-yl]hydrazono $\}-3$-substituted-1,3- thiazolidin-4- one derivatives (5a-f). Whereas, products (5) were obtained by reacting (3) with 2 chloroacetyl chloride (Scheme 2).

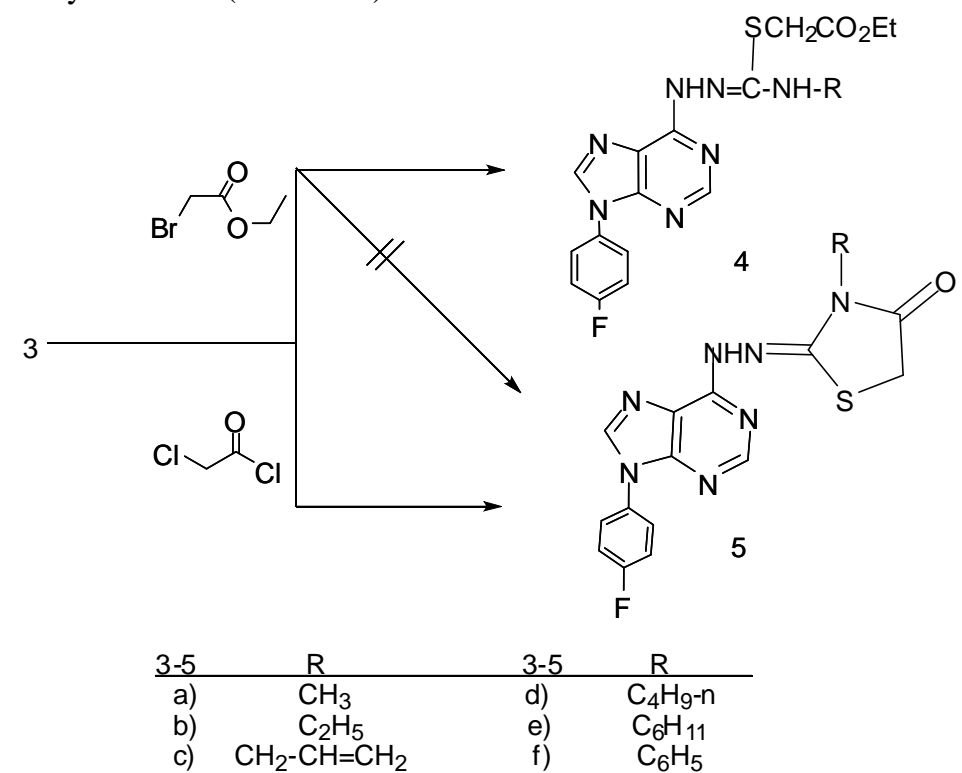

Scheme 2.

Egypt. J. Chem. 56, No. 1 (2013) 
IR spectra of products (4) showed $v_{\mathrm{C}=\mathrm{O}}$ (ester) at $1710-1744 \mathrm{~cm}^{-1}$ region; beside the other characteristic absorption peaks. Moreover, their ${ }^{1} \mathrm{H}-\mathrm{NMR}$ spectra revealed presence of ethyl proton signals at $\delta: 0.80-1.18$ (triplet) and 3.75-3.90 ppm for $\mathrm{CH}_{3}$ and $\mathrm{CH}_{2}$, respectively.

${ }^{1} \mathrm{H}-\mathrm{NMR}$ spectra of the thiazolidin-4-one derivatives (5) showed the expected $\mathrm{CH}_{2}$ (thiazolidinone) proton signals at $\delta: 3.95-4.05$ ppm region ${ }^{(20)}$; beside the characteristic aromatic proton signals.

The present work was extended to probe the reactivity of 1-(9-(4-fluorophenyl)$9 H$-purin-6-yl)hydrazine (2a) towards some aldopentoses or aldohexsose targeting to synthesize newer acyclo $C$-nucleosides, having 4-fluorophenyl moiety at 9-positon of the purine ring in their structure, of potential useful biological activity.

Thus, condensation of the hydrazine (2a) with the aldopentose (6a) or with aldohexoses (6b-d) gave the corresponding 5-\{[9-(4-fluoro- phenyl)-9H-purin6-yl]hydrazono $\}$ pentane-1,2,3,4-tetrol (7a) and 6-\{[9-(4-fluorophenyl)-9H-purin6-yl]hydrazono \}hexane-1,2,3,4,5-pentols (7b-d) in good yields (Scheme 3).
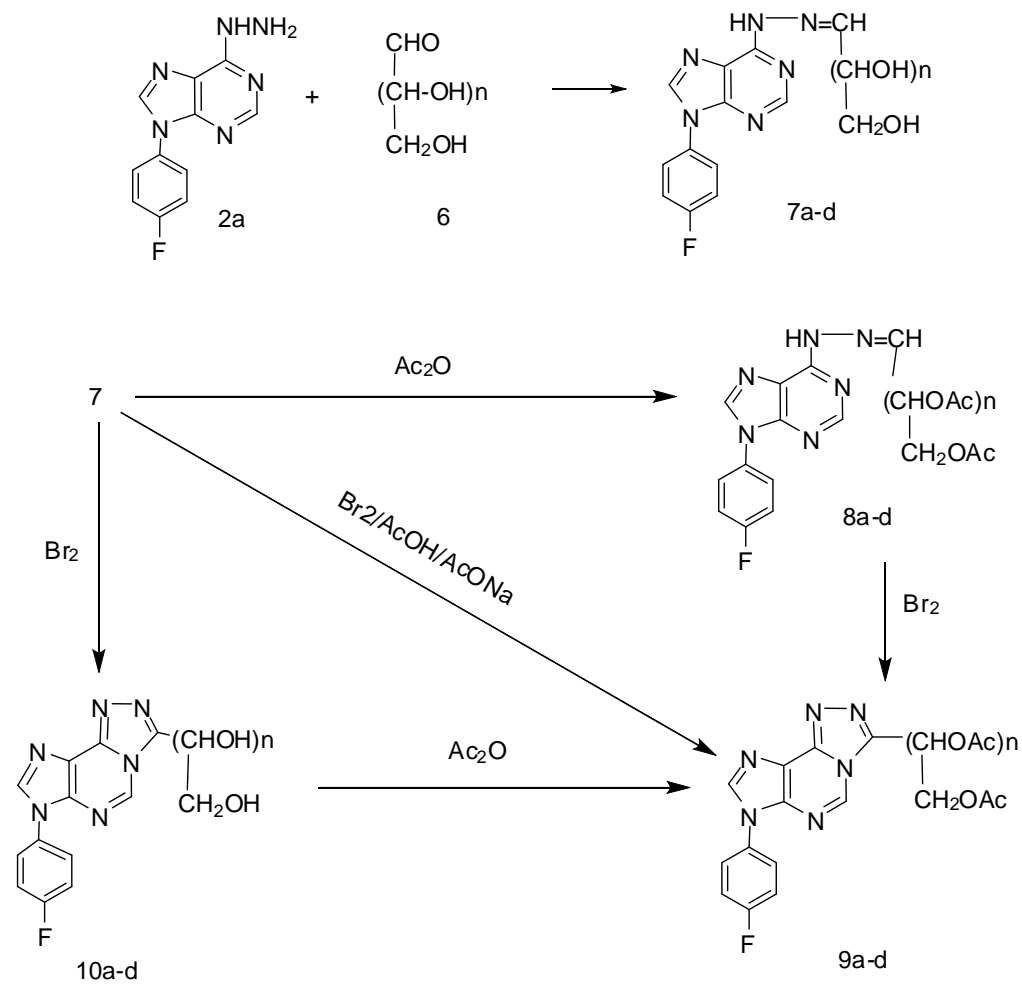

6-10: a) $n=3, \quad b-d) n=4$.
a) D-Arabinose
b) D-Glucose
c) D-Galactose
d) D-Mannose

Scheme 3.

Egypt. J. Chem. 56, No. 1 (2013) 
IR spectra of products (7) showed absorptions due to $\mathrm{C}=\mathrm{N}$ group at 1664$1622 \mathrm{~cm}^{-1}$ region. Their ${ }^{1} \mathrm{H}-\mathrm{NMR}$ showed signals for $\mathrm{CH}_{2}$ and $\mathrm{CH}$ (sugar) around 3.20-5.00 ppm, and showed $\mathrm{OH}$ protons signals of the sugar chain of most of the obtained hydrazones as broad signals at $\delta: 4.6,5.3$ and $\delta: 6.30 \mathrm{ppm}$ together with another $\mathrm{OH}$ and another broad signal at $\delta: 9.05 \mathrm{ppm}\left(\mathrm{D}_{2} \mathrm{O}\right.$-exchangeable). Also, the spectra showed characteristic signals due to azo-methine $(\mathrm{CH}=\mathrm{N}-)$ at $\delta$ : $7.75 \mathrm{ppm}$; or together with aromatic protons at $7.45 \mathrm{ppm}$. All the ${ }^{1} \mathrm{H}-\mathrm{NMR}$ spectra of the hydrazones 7 showed $\mathrm{H}-8$ and $\mathrm{H}-2$ of the purine ring as sharp signals around $\delta: 8.25-8.35 \mathrm{ppm}$ region and at $8.65 \mathrm{ppm}$. Mass spectra of the hydrazones $7 \mathrm{~b}$ and d showed low intensity $\mathrm{M}^{+1}$ peak at $\mathrm{m} / z$ at 406 (RI, 2.29 and $2.77 \%$, respectively). Low intensity peak signals of some similar compounds have been previously reported ${ }^{21}$ for some aldoses: (6-methyl-4-oxo-2pyrimidinyl)-hydrazones.

Acetylation of the hydrazones (7a-d) with acetic anhydride in the presence of pyridine at room temperature gave the acetic acid 2,3,4-triacetoxy-5-\{[9-(4fluoro-phenyl)-9H-purin-6-yl]-hydrazono\}pentyl ester (8a) and acetic acid 2,3,4,5- tetraacetoxy-6- \{[9-(4-fluoro-phenyl)- 9H-purin-6-yl]- hydrazono $\}$ hexyl esters (8b-d) (Scheme 4).

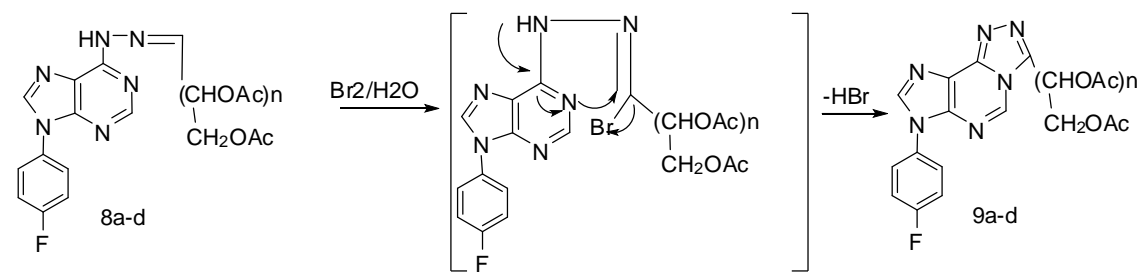

Scheme 4.

IR absorption spectra of the latter compounds showed the expected OAc and $\mathrm{C}=\mathrm{N}$ groupings absorptions. Their ${ }^{1} \mathrm{H}-\mathrm{NMR}$ spectra revealed signals of four $\mathrm{O}$ acetyl (for pentose derivative (8a) or five $\mathrm{O}$-acetyl groups (for hexose derivatives (8b-d), respectively. Mass spectrum for (8a) showed an $\mathrm{M}^{+}$peak at $\mathrm{m} / \mathrm{z}$ 545(1.88\%); while the spectra of products (8b) and (8d) showed peaks at $m / z$ : $616(36.07 \%), 616(0.72 \%)$, respectively.

Oxidative cyclization of products (7a-d) with bromine in acetic acid in the presence of anhydrous sodium acetate afforded 1-[7-(4-fluorophenyl)-7H[1,2,4]triazolo[3,4-i]purin-3-yl]butane-1,2,3,4-tetra-acetate (9a) and 1-[7-(4fluorophenyl)-7H-[1,2,4]triazolo[3,4-i]purin-3-yl]pentane-1,2,3,4,5-penta acetate derivatives (9b-d). The latter products were obtained also by oxidative cyclization of the hydrazone acetates (9a-d) upon reacting with bromine water while stirring at room temperature (m.p. and mixed m.p. gave no depression). The following schematic pathway can explain cyclization of the hydrazone acetates (8) to their corresponding triazolo-derivatives (9): 
IR spectra of products (9) showed generally the characteristic $\mathrm{C}=\mathrm{O}$ (acetyl) and $\mathrm{C}=\mathrm{N}$ absorptions around 1750 and $1620 \mathrm{~cm}^{-1}$ regions, respectively. Also, their ${ }^{1} \mathrm{H}-\mathrm{NMR}$ spectra revealed $\mathrm{CH}_{3}$ (acetyl) proton signals around $\delta: 1.7-2.4$ ppm, and signals for $\mathrm{CH}_{2}, \mathrm{CH}$ (multiplet) and aromatic proton signals, around $\delta$ : 4.10-4.48, 5.10-5.59 and 7.40-7.90 ppm regions, respectively. The spectra included also H-8 and H-5 proton signals of the purine nucleus around $\delta$ : 8.378.84 and 8.63-8.95 ppm regions, respectively. Mass spectrum of product (9a) showed $\left(\mathrm{M}^{+}\right) \mathrm{m} / \mathrm{z}$ peak at: $542(0.88 \%)$, and the spectra of products ( $\left.9 \mathrm{~b}-\mathrm{d}\right)$ showed $\mathrm{m} / \mathrm{z}$ peaks at $614(14,0.37$, and $0.60 \%$, respectively.

Oxidative cyclization of products (7a-d) with bromine/water at room temperature afforded smoothly the 1-[7-(4-fluorophenyl)-7H-[1,2,4]triazolo[3,4-i]purin-3-yl]butane-1,2,3,4-tetrol (10a) and 1-[7-(4-fluoro-phenyl)-7 $\mathrm{H}$ $[1,2,4]$ triazolo[3,4-i]purin-3-yl]pentane-1,2,3,4,5-pentols (10b-d).

Treatment of products (10) with acetic anhydride in pyridine gave readily the products $(9 \mathrm{a}-\mathrm{d})$ in good yield.

${ }^{1} \mathrm{H}-\mathrm{NMR}$ spectrum of product (10b) revealed the $\mathrm{CH}_{2}$ proton associated with solvent $\left(\right.$ DMSO- $\left.\mathrm{d}_{6}\right)$ as broad signals around $\delta: 3.20-3.50 \mathrm{ppm}$.

\section{Experimental}

All melting points are uncorrected. Microanalyses were carried out by the Microanalytical Laboratory, National Research Center, Cairo, Egypt. Infrared spectra (KBr-disc) were recorded using a Jasco FT/IR-300E spectrophotometer. ${ }^{1} \mathrm{H}$ NMR spectra were recorded in DMSO- $\mathrm{d}_{6}$ using Varian mercury $300 \mathrm{MHz}$ and Varian Gemini $200 \mathrm{MHz}$ with chemical shift in $\delta$ from $\mathrm{Me}_{4} \mathrm{Si}$. Mass spectra were recorded on GC/MS finnigan SSQ 7000 spectrophotometer.

9-(4-Fluorophenyl)-6-hydrazino-9H-purine (2a) and 9-(4-fluoro-phenyl)-6-(1methylhydrazino)-9H-purine $(2 b)$

General procedure

A mixture of the chloropurine 1 (0.01 mol.) and hydrazine hydrate or methylhydrazine (5ml) in absolute ethanol was heated under reflux for $1 \mathrm{hr}$.The solid product obtained was filtered off and recrystallized from ethanol to give 2 .

(2a): yield $75 \%$, m.p. $238-240^{\circ} \mathrm{C}$. IR (KBr) v: $3350,3300,3178$ (NH). Mass $(\mathrm{m} / \mathrm{z}, \%): 244\left(\mathrm{M}^{+}, 0.88\right)$.

Anal. Calc. $\mathrm{C}_{11} \mathrm{H}_{9} \mathrm{FN}_{6}$ (244.23): C, 54.10, H, 3.71, F, 7.78, N, 34.41. Found: C, 54.00, H, 3.75, F, 7.75, N, $34.40 \%$.

(2b): yield 74\%, m.p. $218-220^{\circ} \mathrm{C} . \mathrm{IR}(\mathrm{KBr})$ v: $3260(\mathrm{NH}) .{ }^{1} \mathrm{H}-\mathrm{NMR} \delta: 3.92$ (s, 5H. $\left.\mathrm{CH}_{3}, \mathrm{NH}_{2}\right) ; 7.50(\mathrm{~m}, 2 \mathrm{H}, 2 \mathrm{CH}(\mathrm{Ar})) ; 7.87(\mathrm{~m}, 2 \mathrm{H}, 2 \mathrm{CH}(\mathrm{Ar})) ; 8.42(\mathrm{~s}, 1 \mathrm{H}$, $\mathrm{H}-8) ; 8.81$ (s, 1H, H-2). Mass (m/z, \%): $258\left(\mathrm{M}^{+}, 92.51\right)$.

Egypt. J. Chem. 56, No. 1 (2013) 
Anal. Calc. $\mathrm{C}_{12} \mathrm{H}_{11} \mathrm{FN}_{6}$ (258.25): C, 55.81, H, 4.29, F, 7.36, N, 32.54. Found: C, 55.80, H, 4.25, F, 7.35, N, 32.50\%.

2-[9-(4-fluorophenyl)-9H-purin-6-yl]-N-substitutedhydrazine-carbo- thioamides $(3 a-f)$

General procedure

A mixture of $2 \mathrm{a}(0.01 \mathrm{~mol})$ and the appropriate isothiocyanate $(0.012 \mathrm{~mol}$.) in absolute ethanol $(40 \mathrm{ml})$ was heated under reflux for $3 \mathrm{hr}$. After cooling, the solid product obtained was filtered off, washed with ethanol, dried and crystallized from ethanol to give $3 \mathrm{a}-\mathrm{f}$.

2-[9-(4-Fluorophenyl)-9H-purin-6-yl]-N-methylhydrazinecarbothio-amide (3a): yield 77\%, m.p. $224-226^{\circ} \mathrm{C} . \mathrm{IR}(\mathrm{KBr})$ v: $3231,3166(\mathrm{NH}) ; 1295(\mathrm{C}=\mathrm{S}) .{ }^{1} \mathrm{H}-\mathrm{NMR}$ $\delta: 2.85\left(\mathrm{~s}, 3 \mathrm{H} . \mathrm{CH}_{3}\right) ; 7.45(\mathrm{~m}, 2 \mathrm{H}, 2 \mathrm{CH}(\mathrm{Ar})) ; 7.91(\mathrm{~m}, 2 \mathrm{H}, 2 \mathrm{CH}(\mathrm{Ar})) ; 7.95$ (b,1H,NH ); 8.37 (s,1H, H-8 ); 8.65 (s, 1H, H-2) ; 9.40 (b, 1H, NH) ; 9.73 (b,1H, $\mathrm{NH}$ ).

Anal. Calc. $\mathrm{C}_{13} \mathrm{H}_{12} \mathrm{FN}_{7} \mathrm{~S}$ (317.34): C, 49.20, H, 3.81, F, 5.99, N, 30.90, S, 10.10.Found: C, 49.00, H, 3.75, F, 6.00, N, 30.50, S, $9.85 \%$.

N-Ethyl-2-[9-(4- fluorophenyl) -9H-purin-6-yl] hydrazinecarbo- thioamide (3b): yield 74\%, m.p. 190-192 ${ }^{\circ}$ C.IR (KBr) v: 3233, $3169(\mathrm{NH}) ; 1295(\mathrm{C}=\mathrm{S}) .{ }^{1} \mathrm{H}-$ NMR $\delta: 1.05\left(\mathrm{t}, 3 \mathrm{H}, J=7.0 \mathrm{~Hz}, \mathrm{CH}_{3}\right) ; 3.50\left(\mathrm{q}, 2 \mathrm{H}, J=7.0 \mathrm{~Hz}, \mathrm{CH}_{2}\right), 7.50(\mathrm{~m}, 2 \mathrm{H}$, $2 \mathrm{CH}(\mathrm{Ar})) ; 7.87$ (m, 2H, 2CH(Ar)); 8.10 (b, 1H, NH), 8.35 (s, 1H, H-8); 8.70 (s, 1H, H-2) ; 9.45 (b, 1H, NH); 9.70 (b, 1H, NH). Mass (m/z, \%): $331\left(\mathrm{M}^{+}, 9.15\right)$.

Anal. Calc. $\mathrm{C}_{14} \mathrm{H}_{14} \mathrm{FN}_{7} \mathrm{~S}$ (331.37): C, 50.74, H, 4.26, F, 5.73, N, 29.59, S, 9.68. Found: C, 50.60, H, 4.15, F, 5.70, N, 29.50, S, $9.65 \%$.

2-[9-(4-Fluorophenyl) -9H- purin -6- yl] - N- (prop -2- en -1-yl) hydrazinecarbothioamide (3c): yield $71 \%$, m.p. $191-193^{\circ} \mathrm{C} . \mathrm{IR}(\mathrm{KBr})$ v: $3483,3190(\mathrm{NH})$. ${ }^{1} \mathrm{H}-\mathrm{NMR} \delta: 4.10\left(\mathrm{~m}, 2 \mathrm{H}, \mathrm{NCH}_{2}\right), 5.10\left(2 \mathrm{~d}, 2 \mathrm{H}, \mathrm{C}=\mathrm{CH}_{2}\right) ; 5.80(\mathrm{~m}, 1 \mathrm{H}, \mathrm{CH}$ (allyl)); 7.50 (m,2H, 2CH (Ar)); 7.90 (m, 2H, 2CH (Ar)); 8.25 (b, 1H, NH); 8.40 (s,1H, H-8); 8.70 (s, 1H, H-2 ); 9.50 (b, 1H, NH); 9.85 (b, 1H, NH). Mass (m/z, $\%): 343\left(\mathrm{M}^{+}, 6.12\right)$.

Anal. Calc. $\mathrm{C}_{15} \mathrm{H}_{14} \mathrm{FN}_{7} \mathrm{~S}$ (343.38): C, 52.47, H, 4.11, F, 5.53, N, 28.55, S, 9.34. Found: C, 52.35, H, 4.25, F, 5.50, N, 28.45, S, 9.45\%.

$N$ - (n-Butyl) -2-[9-(4-fluorophenyl)-9H-purin-6-yl]hydrazinecarbothio-amide (3d): yield $75 \%$, m.p. $181-183^{\circ} \mathrm{C}$ (methanol).IR (KBr) v: $3168(\mathrm{NH})$. Anal. Calc. $\mathrm{C}_{16} \mathrm{H}_{18} \mathrm{FN}_{7} \mathrm{~S}$ (359.42): C, 53.47, H, 5.05, F, 5.29, N, 27.28, S, 8.92. Found: C, 53.45, H, 5.00, F, 5.30, N, 27.30, S, 8.95\%.

$\mathrm{N}$ - (Cyclohexyl) -2- [9- (4-fluorophenyl) - 9H - purin -6- yl] hydrazinecarbothioamide (3e): yield $63 \%$, m.p. $227-229^{\circ} \mathrm{C}$ (methanol). IR (KBr) v: $3180(\mathrm{NH})$. Mass (m/z, \%): $385\left(\mathrm{M}^{+}, 17\right)$.

Anal. Calc. $\mathrm{C}_{18} \mathrm{H}_{20} \mathrm{FN}_{7} \mathrm{~S}$ (385.46): C, 56.09, H, 5.23, F, 4.93, N, 25.44, S, 8.32. Found: C, 55.95, H, 5.45, F, 4.90, N, 25.55, S, 8.45\%. 
2-[9-(4-Fluorophenyl)-9H-purin-6-yl]-N-phenylhydrazinecarbothio- amide (3f): yield 64\%, m.p. $207-209^{\circ} \mathrm{C}$ (DMF).IR (KBr) v: $3186(\mathrm{NH})$.

Anal. Calc. $\mathrm{C}_{18} \mathrm{H}_{14} \mathrm{FN}_{7} \mathrm{~S}$ (379.41): C, 56.98, H, 3.72, F, 5.01, N, 25.84, S, 8.45. Found: C, 56.70, H, 3.70, F, 5.00, N, 25.80, S, $8.50 \%$.

Ethyl \{[\{[9-(4-fluorophenyl) -9H-6-yl] hydrazono\} (substitutedamino)- ethyl] sulfanyl) acetates $(4 a-f)$

General procedure

To a suspension of (3a-f) $(0.01 \mathrm{~mol}$.) in absolute ethanol $(50 \mathrm{ml})$, it was added ethyl bromoacetate $(0.01 \mathrm{~mol}$.) and sodium acetate $(0.01 \mathrm{~mol}$.). The reaction mixture was heated under reflux for $3 \mathrm{hr}$ and left to cool. The solid product obtained was filtered off, washed with ethanol, dried and then crystallized from the proper solvent to give product (4a-f).

Ethyl\{[\{[9-(4-fluorophenyl)-9H-6-yl]hydrazono\}(methylamino)ethyl]-sulfanyl\} acetate (4a): yield 63\%, m.p. 227-229 ${ }^{\circ} \mathrm{C}$ (ethanol).IR (KBr) v: $3308(\mathrm{NH}) ; 1746$ $(\mathrm{C}=\mathrm{O})$. Mass $(\mathrm{m} / \mathrm{z}, \%): 403\left(\mathrm{M}^{+}, 2.08\right)$.

Anal. Calc. $\mathrm{C}_{17} \mathrm{H}_{18} \mathrm{FN}_{7} \mathrm{O}_{2} \mathrm{~S}$ (403.43): C, 50.61, H, 4.50, F, 4.71, N, 24.30, S, 7.95. Found: C, 50.80, H, 4.45, F, 4.55, N, 24.25, S, 7.85\%.

Ethyl \{[(ethylamino)\{[9-(4-fluorophenyl)-9H-purin-6-yl]hydrazono\}- methyl] sulfanyl) acetate (4b): yield $79 \%$, m.p. $248-250^{\circ} \mathrm{C}$ (methanol). IR $(\mathrm{KBr})$ v: 3295 , $3124(\mathrm{NH}) ; 1709(\mathrm{C}=\mathrm{O}) .{ }^{1} \mathrm{H}-\mathrm{NMR} \delta: 1.18\left(\mathrm{~m}, 6 \mathrm{H}, 2 \mathrm{CH}_{3}\right) ; 3.30\left(\mathrm{~s}, 2 \mathrm{H}, \mathrm{SCH}_{2}\right)$; $3.90\left(\mathrm{~m}, 4 \mathrm{H}, 2 \mathrm{CH}_{2}\right) ; 7.40$ (m, 2H, 2CH(Ar) ); 7.80 (m, 3H, 2CH(Ar), H-8); 8.3 ( s, 1H, H-2); $11.40(\mathrm{~s}, 1 \mathrm{H}, \mathrm{NH})$.

Anal. Calc. $\mathrm{C}_{18} \mathrm{H}_{20} \mathrm{FN}_{7} \mathrm{O}_{2} \mathrm{~S}$ (417.46): C, 51.79, H, 4.83, F, 4.55, N, 23.49, S, 7.68. Found: C, 51.75, H, 4.85, F, 4.50, N, 23.50, S, 7.65\%.

Ethyl \{[\{[9-(4-fluorophenyl) -9H-purin-6-yl] hydrazono\} (prop-2-en-1ylamino) methyl] sulfanyl\} acetate (4c): yield $79 \%$, m.p. $237-239^{\circ} \mathrm{C}$ (methanol). IR (KBr) v: $3355(\mathrm{NH}) ; 1734(\mathrm{C}=\mathrm{O})$.

Anal. Calc. $\mathrm{C}_{19} \mathrm{H}_{20} \mathrm{FN}_{7} \mathrm{O}_{2} \mathrm{~S}$ (429.47): C, 53.14, H, 4.69, F, 4.42, N, 22.83, S, 7.47. Found: C, 53.15, H, 4.55, F, 4.40, N, 22.85, S, 7.40.

Ethyl \{[(n-butylamino) \{[9-(4-fluorophenyl) - 9H- purin -6-yl] hydrazono\}methyl] sulfanyl\} acetate (4d): yield $61 \%$, m.p. $235-237^{\circ} \mathrm{C}$ (methanol). IR (KBr) v: $3316(\mathrm{NH}) ; 1744(\mathrm{C}=\mathrm{O}) .{ }^{1} \mathrm{H}-\mathrm{NMR} \delta: 0.8\left(\mathrm{t}, 3 \mathrm{H}, \mathrm{J}=7.50 \mathrm{~Hz}, \mathrm{CH}_{3}\right) ; 1.3(\mathrm{~m}$, $\left.5 \mathrm{H}, \mathrm{CH}_{2}, \mathrm{CH}_{3}\right) ; 1.60\left(\mathrm{~m}, 2 \mathrm{H}, \mathrm{CH}_{2}\right) ; 3.75\left(\mathrm{~m}, 2 \mathrm{H}, \mathrm{SCH}_{2}\right) ; 4.00$ (s, 2H, N-CH $\mathrm{CH}_{2}$; 4.30 (q, 2H, J=3.5, $\left.\mathrm{CH}_{2}\right) ; 7.60$ (m, 2H, 2CH(Ar)); 7.8 (m, 3H, 2CH(Ar), H-8); 8.4 (s, 1H,H-2 ); $9.80(\mathrm{~s}, 1 \mathrm{H}, \mathrm{NH}) ; 12.30(\mathrm{~s}, 1 \mathrm{H}, \mathrm{NH})$.

Anal. Calc. $\mathrm{C}_{20} \mathrm{H}_{24} \mathrm{FN}_{7} \mathrm{O}_{2} \mathrm{~S}$ (445.51): C, 53.92, H, 5.43, F, 4.26, N, 22.01, S, 7.20. Found: C, 53.90, H, 5.45, F, 4.25, N, 22.05, S, 7.15\%.

Ethyl \{[(cyclohexylamino)\{[9-(4-fluorophenyl)-9H-purin-6-yl]hydra- zono\} methyl] sulfanyl\} acetate (4e): yield $66 \%$, m.p. $265-2267^{\circ} \mathrm{C}$ (methanol). IR (KBr) v: $3310,3180(\mathrm{NH}) ; 1722(\mathrm{C}=\mathrm{O})$. 
Anal. Calc. $\mathrm{C}_{22} \mathrm{H}_{26} \mathrm{FN}_{7} \mathrm{O}_{2} \mathrm{~S}$ (471.55): C, 56.04, H, 5.56, F, 4.03, N, 20.79, S, 6.80. Found: C, 56.05, H, 5.55, F, 4.00, N, 20.80, S, $6.75 \%$.

Ethyl \{[\{[9- (4- fluorophenyl)- 9H-purin - 6- yl] hydrazono\} (phenylamino) methyl] sulfanyl\} acetate (4f): yield $79 \%$, m.p. $248-250^{\circ} \mathrm{C}$ (methanol). IR (KBr) v: 3292, $3200(\mathrm{NH}) ; 1730(\mathrm{C}=\mathrm{O})$.

Anal. Calc. $\mathrm{C}_{22} \mathrm{H}_{20} \mathrm{FN}_{7} \mathrm{O}_{2} \mathrm{~S}$ (465.50): C, 56.76, H, 4.33, F, 4.08, N, 21.06, S, 6.89. Found: C, 56.95, H, 4.15, F, 4.20, N, 21.30, S, $6.65 \%$.

2 - \{[9- (4- Fluorophenyl) - 9H- purin- 6- yl]hydrazono\}- 3- substituted - 1,3thiazolidin -4- ones $(5 a-f)$

General procedure

To a suspension of (3a-f) $(0.01 \mathrm{~mol})$ in methylene chloride $(20 \mathrm{ml})$ it was added chloroacetyl chloride $(0.01 \mathrm{~mol})$ and the resulting mixture was stirred at room temperature for $2 \mathrm{hr}$. The solid product which separated out was filtered off, and crystallized to give (5a-f).

2-\{[9-(4-Fluorophenyl)-9H-purin-6-yl]hydrazono\}-3-methyl-1,3- thiazolidin4-one (5a): yield $80 \%$, m.p. $260-262^{\circ} \mathrm{C}$ (methanol). IR (KBr) v: $3117(\mathrm{NH})$; $1720(\mathrm{C}=\mathrm{O})$.

Anal. Calc. $\mathrm{C}_{15} \mathrm{H}_{12} \mathrm{FN}_{7} \mathrm{OS}$ (357.37): C, 50.41, H, 3.38, F, 5.32, N, 27.44, S, 78.97. Found: C, 50.25, H, 3.35, F, 5.50, N, 27.40, S, 9.00\%.

3-Ethyl-2-\{[9-(4-fluorophenyl)-9H-purin-6-yl]hydrazono\}-1,3-thiazolidin- 4-one (5b): yield $89 \%$, m.p. $248-250^{\circ} \mathrm{C}$ (methanol). IR (KBr) v: $3120(\mathrm{NH}) ; 1720$ $(\mathrm{C}=\mathrm{O}) .{ }^{1} \mathrm{H}-\mathrm{NMR} \delta: 1.20\left(\mathrm{t}, 3 \mathrm{H}, \mathrm{J}=7.0 \mathrm{~Hz}, \mathrm{CH}_{3}\right.$ (ethyl) $) ; 3.90\left(\mathrm{q}, 2 \mathrm{H}, \mathrm{J}=7.0 \mathrm{~Hz},\left(\mathrm{CH}_{2^{-}}\right.\right.$ ethyl)); $4.05\left(\mathrm{~S}, 2 \mathrm{H}, \mathrm{CH}_{2}\right) ; 7.5(\mathrm{~m}, 2 \mathrm{H}, 2 \mathrm{CH}, \mathrm{Ar}) ; 7.85(\mathrm{~m}, 2 \mathrm{H}, 2 \mathrm{CH}, \mathrm{Ar}) ; 8.20$ $(\mathrm{s}, 1 \mathrm{H}, \mathrm{H}-8) ; 8.70(\mathrm{~s}, 1 \mathrm{H}, \mathrm{H}-2)$. Mass (m/z, \%): $370\left(\mathrm{M}^{+}-1,100\right)$.

Anal. Calc. $\mathrm{C}_{16} \mathrm{H}_{14} \mathrm{FN}_{7} \mathrm{OS}$ (371.39): C, 51.74, H, 3.80, F, 5.12, N, 26.40, S, 78.63. Found: C, 51.70, H, 3.70, F, 5.10, N, 26.50, S, 8.55\%.

2- \{[9- (4-Fluorophenyl) -9H-purin -6-yl] hydrazono\}-3-(prop-2-en-1-yl)1,3-thiazolidin-4-one (5c): yield $90 \%$, m.p. $200-202^{\circ} \mathrm{C}$ (acetonitrile). IR (KBr) v: $3142(\mathrm{NH}) ; 1714(\mathrm{C}=\mathrm{O}) .{ }^{1} \mathrm{H}-\mathrm{NMR} \delta: 0.8\left(\mathrm{t}, 3 \mathrm{H}, J=7.50 \mathrm{~Hz}, \mathrm{CH}_{3}\right) ; 1.30(\mathrm{~m}, 5 \mathrm{H}$, $\left.\mathrm{CH}_{2}, \mathrm{CH}_{3}\right) ; 1.60\left(\mathrm{~m}, 2 \mathrm{H}, \mathrm{CH}_{2}\right) ; 3.75\left(\mathrm{~m}, 2 \mathrm{H}, \mathrm{SCH}_{2}\right) ; 4.00\left(\mathrm{~s}, 2 \mathrm{H}, \mathrm{N}-\mathrm{CH}_{2}\right) ; 4.30$ (q, 2H, J=3.5, $\left.\mathrm{CH}_{2}\right) ; 7.60$ ( m, 2H, 2CH(Ar)); 7.8 ( m, 3H, 2CH(Ar), H-8); 8.40 (S, 1H,H-2); $9.80(\mathrm{~s}, 1 \mathrm{H}, \mathrm{NH}) ; 12.30(\mathrm{~s}, 1 \mathrm{H}, \mathrm{NH})$.

Anal. Calc. $\mathrm{C}_{17} \mathrm{H}_{14} \mathrm{FN}_{7} \mathrm{OS}$ (383.40): C, 53.26, H, 3.68, F, 4.96, N, 25.57, S, 8.36. Found: C, 53.25, H, 3.65, F, 5.00, N, 25.55, S, 8.35\%.

3-(n-Butyl)-2-\{[9-(4-fluorophenyl)-9H-purin-6-yl]hydrazono\}-1,3-thiazolidin4-one (5d): yield $90 \%$, m.p. $175-177^{\circ} \mathrm{C}$ (methanol). IR (KBr) v: $3320(\mathrm{NH})$; $1758(\mathrm{C}=\mathrm{O})$.

Anal. Calc. $\mathrm{C}_{18} \mathrm{H}_{18} \mathrm{FN}_{7} \mathrm{OS}$ (399.45): C, 54.12, H, 4.54, F, 4.76, N, 24.50, S, 8.03. Found: C, 54.15, H, 4.50, F, 4.75, N, 24.55, S, 8.00\%. 
3-Cyclohexyl-2-\{[9-(4-fluorophenyl)-9H-purin-6-yl]hydrazono\}-1,3-thiazolidin4-one (5e): yield 80\%, m.p. $197-199^{\circ} \mathrm{C}$ (methanol). IR (KBr) v: $3118(\mathrm{NH}) ; 1730$ $(\mathrm{C}=\mathrm{O})$. Mass $(\mathrm{m} / \mathrm{z}, \%): 425\left(\mathrm{M}^{+}, 97\right)$.

Anal. Calc. $\mathrm{C}_{20} \mathrm{H}_{20} \mathrm{FN}_{7} \mathrm{OS}$ (425.48): C, 56.46, H, 4.75, F, 4.47, N, 23.04, S, 7.54. Found: C, 56.40, H, 4.95, F, 4.50, N, 23.50, S, 7.50\%.

2-\{[9-(4-Fluorophenyl)-9H-purin-6-yl]hydrazono\}-3-phenyl-1,3-thiazolidin-

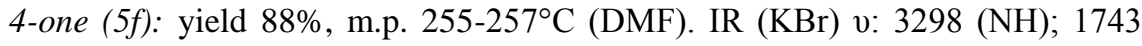
$(\mathrm{C}=\mathrm{O})$.

Anal. Calc. $\mathrm{C}_{20} \mathrm{H}_{14} \mathrm{FN}_{7} \mathrm{OS}$ (419.43): C, 57.27, H, 3.36, F, 4.53, N, 23.38, S, 7.64. Found: C, 57.45, H, 3.40, F, 4.30, N, 23.45, S, 7.50\%.

5- \{[9-(4-Fluorophenyl) -9H- purin-6-yl] hydrazono\} pentane -1,2,3,4-tetrol (7a) and 6-\{[9-(4-fluorophenyl)-9H-purin-6-yl]hydrazono\}-hexane-1,2,3,4,5-pentols $(7 b-d)$

General procedure

To a solution of (2a) $(0.005 \mathrm{~mol})$ in ethanol $(30 \mathrm{ml})$, the appropriate sugar $(0.005 \mathrm{~mol})$ in water $(2 \mathrm{ml})$ was added. The reaction mixture was heated on a steam-bath for $15 \mathrm{~min}$ and left over night at room temperature; the crystalline product which separated out was filtered, washed with ethanol, and recrystallized from water-ethanol to give (7a-d).

5-\{[9-(4-Fluorophenyl)-9H-purin-6-yl]hydrazono\}pentane-1,2,3,4-tetrol (7a): yield $78 \%$, m.p. $190-192^{\circ} \mathrm{C}$ (aq. ethanol). IR (KBr) v: 3400, 3241 (NH); 1652 $(\mathrm{C}=\mathrm{N})$.

Anal. Calc. $\mathrm{C}_{16} \mathrm{H}_{17} \mathrm{FN}_{6} \mathrm{O}_{4}$ (376.34): C, 51.06, H, 4.55, F, 5.05, N, 22.33. Found: C, 51.20, H, 4.35, F, 5.20, N, 22.45\%.

6-\{[9-(4-Fluorophenyl)-9H-purin-6-yl]hydrazono\}hexane-1,2,3,4,5-pentaol (7b): yield $80 \%$, m.p. $190-192^{\circ} \mathrm{C}$ (aq. ethanol). IR (KBr) v: $3300,3200(\mathrm{NH}) ; 1664$ $\mathrm{C}=\mathrm{N}) .{ }^{1} \mathrm{H}-\mathrm{NMR} \delta: 3.25\left(\mathrm{~m}, 2 \mathrm{H}, \mathrm{CH}_{2}\right) ; 3.50(\mathrm{~m}, 2 \mathrm{H}, 2 \mathrm{CH}) ; 3.70(\mathrm{~m}, 1 \mathrm{H}, \mathrm{CH})$; 3.95( $\mathrm{m}, 1 \mathrm{H}, \mathrm{CH}) ; 4.40$ ( b, 1H, OH ); 4.95 ( b, 2H, 2OH ); 6.15 (b, 2H, 2OH ); 7.45(m, 3H, 2CH(Ar)), N=CH ); $7.90(\mathrm{~m}, 2 \mathrm{H}, 2 \mathrm{CH}(\mathrm{Ar})) ; 8.25$ ( s, 1H, H-8 ); 8.65 ( s, 1H, H-2); 9.05 ( b, 1H, NH ). Mass (m/z, \%): 406( $\left.\mathrm{M}^{+}, 2.25\right)$.

Anal. Calc. $\mathrm{C}_{17} \mathrm{H}_{19} \mathrm{FN}_{6} \mathrm{O}_{4}$ (406.37): C, 50.25, H, 4.71, F, 4.68, N, 20.68 . Found: C, 50.20, H, 4.65, F, 4.66, N, 20.65\%.

6-\{[9-(4-Fluorophenyl)-9H-purin-6-yl]hydrazono\}hexane-1,2,3,4,5-pentaol (7c): yield $73 \%$, m.p. $216-218^{\circ} \mathrm{C}$ (aq. ethanol). IR (KBr) v: 3350, 3254, $3150(\mathrm{NH})$; $1624(\mathrm{C}=\mathrm{N})$.

Anal. Calc. $\mathrm{C}_{17} \mathrm{H}_{19} \mathrm{FN}_{6} \mathrm{O}_{4}$ (406.37): C, 50.25, H, 4.71, F, 4.68, N, 20.68. Found: C, 50.30, H, 4.80, F, 4.60, N, 20.66\%.

6-\{[9-(4-Fluorophenyl)-9H-purin-6-yl]hydrazono\}hexane-1,2,3,4,5-pentaol (7d): yield $76 \%$, m.p. $181-183^{\circ} \mathrm{C}$ (aq. ethanol). IR (KBr) v: 3386, $3250(\mathrm{NH}) ; 1622$ $(\mathrm{C}=\mathrm{N}) .{ }^{1} \mathrm{H}-\mathrm{NMR} \delta: 3.40\left(\mathrm{~m}, 2 \mathrm{H}, \mathrm{CH}_{2}\right) ; 3.60(\mathrm{~m}, 2 \mathrm{H}, 2 \mathrm{CH}) ; 3.70(\mathrm{~m}, 1 \mathrm{H}, \mathrm{CH})$;

Egypt. J. Chem. 56, No. 1 (2013) 
4.20( m, 1H, CH ); 4.40 ( b, 1H, OH ); 4.50 ( b, 2H, 2OH ); 5.30 ( b, 2H, 2OH ); $7.40(\mathrm{~m}, 2 \mathrm{H}, 2 \mathrm{CH}(\mathrm{Ar})) ; 7.75(\mathrm{~s}, 1 \mathrm{H}, \mathrm{N}=\mathrm{CH}) ; 7.95(\mathrm{~m}, 2 \mathrm{H}, 2 \mathrm{CH}(\mathrm{Ar})) ; 8.35(\mathrm{~s}$, $1 \mathrm{H}, \mathrm{H}-8$ ); 8.65 ( $\mathrm{s}, 1 \mathrm{H}, \mathrm{H}-2$ ); $11.50(\mathrm{~b}, 1 \mathrm{H}, \mathrm{NH})$. Mass $(\mathrm{m} / \mathrm{z}, \%): 406\left(\mathrm{M}^{+}\right.$, 2.77).

Anal. Calc. $\mathrm{C}_{17} \mathrm{H}_{19} \mathrm{FN}_{6} \mathrm{O}_{4}$ (406.37): C, 50.25, H, 4.71, F, 4.68, N, 20.68. Found: C, 50.20, H, 4.75, F, 4.65, N, 20.55\%.

Acetic acid 2,3,4- triacetoxy-5- \{[9-(4-fluoro-phenyl) -9H-purin-6-yl]-hydrazono\} butyl ester (8a) and Acetic acid 2,3,4,5-tetraacetoxy-6-\{[9-(4-fluoro-phenyl)-9Hpurin-6-yl]-hydrazono\}pentyl esters $(8 b-d)$ :

General procedure

A solution of (7a-d) $\left(0.004\right.$ mol.) in pyridine $(3 \mathrm{ml})$ was treated with $\mathrm{Ac}_{2} \mathrm{O}$ $(15 \mathrm{ml})$ and left overnight at ambient temperature. The reaction mixture was poured onto ice and the solid product which separated out was filtered off, washd with water and crystallized from ethanol to give (8a-d).

(8a): yield 68\%, m.p. $161-163^{\circ} \mathrm{C}$ (ethanol). IR (KBr) v: $3466(\mathrm{NH}) ; 1748$, $1700(\mathrm{C}=\mathrm{O}) ; 1650(\mathrm{C}=\mathrm{N}) .{ }^{1} \mathrm{H}-\mathrm{NMR} \delta: 1.90-2.10\left(4 \mathrm{~s}, 12 \mathrm{H}, \mathrm{CH}_{3}\right) ; 3.90(\mathrm{~m}, 1 \mathrm{H}$, $\left.\mathrm{CH}_{2}\right) ; 3.90,4.15\left(2 \mathrm{~m}, 2 \mathrm{H}, \mathrm{CH}_{2}\right) ; 5.15(\mathrm{~m}, 1 \mathrm{H}, \mathrm{CH}) ; 5.30(\mathrm{~m}, 1 \mathrm{H}, \mathrm{CH}) ; 5.50(\mathrm{~m}$, $1 \mathrm{H}, \mathrm{CH}) ; 6.55(\mathrm{~s}, 1 \mathrm{H}, \mathrm{N}=\mathrm{CH}) ; 7.50(\mathrm{~m}, 2 \mathrm{H}, 2 \mathrm{CH}(\mathrm{Ar})) ; 7.96(\mathrm{~m}, 2 \mathrm{H}, 2 \mathrm{CH}(\mathrm{Ar})$ ); 9.00 (s, 2H, H-8, H-2 ); 10.55 (b, 1H, NH). Mass (m/z, \%): $545\left(\mathrm{M}^{+}+1,1.88\right)$.

Anal. Calc. $\mathrm{C}_{24} \mathrm{H}_{25} \mathrm{FN}_{6} \mathrm{O}_{8}(544.49)$ : C, 52.94, H, 4.63, F, 3.49, N, 15.43 . Found: C, 52.95, H, 4.65, F, 3.50, N, 15.45\%.

(8b): yield 76\%, m.p. $166-168^{\circ} \mathrm{C}$ (ethanol). IR (KBr) v: $3298(\mathrm{NH}) ; 1726$ $(\mathrm{C}=\mathrm{O}) ; 1624(\mathrm{C}=\mathrm{N}) .{ }^{1} \mathrm{H}-\mathrm{NMR} \delta: 1.60,1.90,2.05,2.25\left(1 \mathrm{~d}, 4 \mathrm{~s}, 15 \mathrm{H}, 5 \mathrm{CH}_{3}\right)$; $3.50\left(\mathrm{~m}, 2 \mathrm{H}, \mathrm{CH}_{2}\right) ; 4.65(\mathrm{~m}, 1 \mathrm{H}, \mathrm{CH}) ; 4.80(\mathrm{~m}, 1 \mathrm{H}, \mathrm{CH}) ; 4.90(\mathrm{~m}, 1 \mathrm{H}, \mathrm{CH})$; $5.30(\mathrm{~m}, 1 \mathrm{H}, \mathrm{CH}) ; 7.00(\mathrm{~d}, 1 \mathrm{H}, \mathrm{N}=\mathrm{CH}) ; 7.50(\mathrm{~m}, 2 \mathrm{H}, 2 \mathrm{CH}(\mathrm{Ar})) ; 7.90(\mathrm{~m}, 2 \mathrm{H}$, $2 \mathrm{CH}(\mathrm{Ar})) ; 8.85(\mathrm{~s}, 1 \mathrm{H}, \mathrm{H}-8) ; 9.00(\mathrm{~s}, 1 \mathrm{H}, \mathrm{H}-2) ; 10.25$ (b, 1H, NH). Mass (m/z, \%): $616\left(\mathrm{M}^{+}, 39.07\right)$.

Anal. Calc. $\mathrm{C}_{27} \mathrm{H}_{29} \mathrm{FN}_{6} \mathrm{O}_{10}$ (616.55): C, 52.60, H, 4.74, F, 3.08, N, 13.63. Found: C, 52.65, H, 4.75, F, 3.05, N, 13.65\%.

(8c): yield $73 \%$, m.p. $170-172^{\circ} \mathrm{C}$ (ethanol). IR (KBr) v: $3466(\mathrm{NH}) ; 1748$, $1720(\mathrm{C}=\mathrm{O}) ; 1652(\mathrm{C}=\mathrm{N}) .{ }^{1} \mathrm{H}-\mathrm{NMR} \delta: 1.90-2.24\left(5 \mathrm{~s}, 15 \mathrm{H}, 5 \mathrm{CH}_{3}\right) ; 3.90,4.10$ $\left(2 \mathrm{~m}, 2 \mathrm{H}, \mathrm{CH}_{2}\right) ; 5.17(\mathrm{~m}, 1 \mathrm{H}, \mathrm{CH}) ; 5.32(\mathrm{~m}, 1 \mathrm{H}, \mathrm{CH}) ; 5.49(\mathrm{~m}, 1 \mathrm{H}, \mathrm{CH}) ; 5.55$ $(\mathrm{m}, 1 \mathrm{H}, \mathrm{CH}) ; 6.60(\mathrm{~d}, 1 \mathrm{H}, \mathrm{N}=\mathrm{CH}) ; 7.60(\mathrm{~m}, 2 \mathrm{H}, 2 \mathrm{CH}(\mathrm{Ar})) ; 7.80(\mathrm{~m}, 2 \mathrm{H}$, $2 \mathrm{CH}(\mathrm{Ar})) ; 9.02$ (s, 2H, H-2, H-8). 10.00 (b, 1H, NH). Mass (m/z, \%): $616\left(\mathrm{M}^{+}\right.$, 0.72).

Anal. Calc. $\mathrm{C}_{27} \mathrm{H}_{29} \mathrm{FN}_{6} \mathrm{O}_{10}$ (616.55): C, 52.60, H, 4.74, F, 3.08, N, 13.63. Found: C, 52.68, H, 4.72, F, 3.00, N, 13.70\%.

(8d): yield 73\%, m.p. $144-146^{\circ} \mathrm{C}$ (methanol). IR (KBr) v: $3446(\mathrm{NH}) ; 1728$, $1694(\mathrm{C}=\mathrm{O}) ; 1650(\mathrm{C}=\mathrm{N})$.

Anal. Calc. $\mathrm{C}_{27} \mathrm{H}_{29} \mathrm{FN}_{6} \mathrm{O}_{10}$ (616.55): C, 52.60, H, 4.74, F, 3.08, N, 13.63. Found: C, 52.55, H, 4.80, F, 3.05, N, 13.65\%. 
1-[7- (4-Fluorophenyl) -7H- [1,2,4]triazolo [3,4-i] purin-3-yl] butane- 1,2,3,4-tetraacetate (9a) and 1-[7-(4-fluorophenyl)-7H-[1,2,4]triazolo-[3,4-i]purin3-yl]pentane-1,2,3,4-pentaacetate derivatives $(9 b-d)$

Method A

To a stirred mixture of the respective $(8 \mathrm{a}-\mathrm{d})(0.003 \mathrm{~mol})$ and anhy. sod. acetate $(5 \mathrm{ml})$ was added bromine $(0.002 \mathrm{~mol}$.) dropwise and the resulting clear solution was stirred for another $2 \mathrm{hr}$. Evaporation of the solvent under reduced pressure gave a residue which was crystallized from ethanol to give (9a-d).

\section{Method B}

A solution of the appropriate $(10 \mathrm{a}-\mathrm{d})(0.003 \mathrm{~mol})$ in pyridine $(5 \mathrm{ml})$ was stirred with acetic acid anhydride $(5 \mathrm{ml})$ for $24 \mathrm{hr}$ at room temperature (ca. 25 $\left.{ }^{\circ} \mathrm{C}\right)$. The reaction mixture was poured onto crushed ice and the solid product was filtered, washed with $\mathrm{H}_{2} \mathrm{O}$ and crystallized from ethanol to give (9a-d).

\section{Method C}

To a mixture of $(7 \mathrm{a}-\mathrm{d})(0.03 \mathrm{~mol})$ and anhy. sod. acetate $(0.03 \mathrm{~mol})$ in glacial acetic acid $(15 \mathrm{ml})$, a solution of bromine $(0.002 \mathrm{~mol}$.) in glacial acetic acid $(5 \mathrm{ml})$ was added and stirred for $1 \mathrm{hr}$ at room temperature $\left(\mathrm{ca} .25^{\circ} \mathrm{C}\right)$. The reaction mixture was treated with acetic acid anhydride $(15 \mathrm{~mol})$ and left over night at room temperature (ca. $25^{\circ} \mathrm{C}$ ), followed by evaporation under reduced pressure. The obtained residue was extracted with chloroform $(2 \times 20 \mathrm{~m} 1)$. Evaporation of the solvent gave a residue which crystallized from ethanol to give ( $9 a-d)$.

(9a): yield 64\%(A), 60\%(B), 68\%(C), m.p. $175-177^{\circ} \mathrm{C}$ (ethanol). IR (KBr) v: $1746(\mathrm{C}=\mathrm{O}) .{ }^{1} \mathrm{H}-\mathrm{NMR} \delta: 1.80-2.20\left(\mathrm{~m}, 12 \mathrm{H}, 4 \mathrm{CH}_{3}\right) ; 4.10,4.25(2 \mathrm{~m}, 2 \mathrm{H}$, $\left.\mathrm{CH}_{2}\right) ; 5.15(\mathrm{~m}, 1 \mathrm{H}, \mathrm{CH}) ; 5.35(\mathrm{~m}, 1 \mathrm{H}, \mathrm{CH}) ; 5.60(\mathrm{~m}, 1 \mathrm{H}, \mathrm{CH}) ; 7.42(\mathrm{~m}, 2 \mathrm{H}$, $2 \mathrm{CH}(\mathrm{Ar})) ; 7.90$ (m, 2H, 2CH(Ar)); 8.37 (s, 1H, H-8); 8.63 (s, 1H, H-5). Mass (m/z, \%): $542\left(\mathrm{M}^{+}, 0.88\right)$.

Anal. Calc. $\mathrm{C}_{24} \mathrm{H}_{23} \mathrm{FN}_{6} \mathrm{O}_{8}$ (542.47): C, 53.14, H, 4.27, F, 3.50, N, 15.49. Found: C, 53.10, H, 4.25, F, 3.40, N, $15.35 \%$.

(9b): yield 74\%(A), 65\%(B), 78\%(C), m.p.206-208 ${ }^{\circ} \mathrm{C}$ (ethanol). IR (KBr) v: $1752(\mathrm{C}=\mathrm{O}) .{ }^{1} \mathrm{H}-\mathrm{NMR} \delta: 1.70-2.27\left(5 \mathrm{~s}, 15 \mathrm{H}, 5 \mathrm{CH}_{3}\right) ; 3.40,3.53\left(\mathrm{~m}, 2 \mathrm{H}, \mathrm{CH}_{2}\right)$; $4.64(\mathrm{~m}, 1 \mathrm{H}, \mathrm{CH}) ; 4.74(\mathrm{~m}, 1 \mathrm{H}, \mathrm{CH}) ; 4.89(\mathrm{~m}, 1 \mathrm{H}, \mathrm{CH}) ; 5.23(\mathrm{~m}, 1 \mathrm{H}, \mathrm{CH}) ; 7.51$ (m, 2H, 2CH(Ar)); 7.94 (m, 2H, 2CH(Ar)); 8.84 (s,1H, H-8); 8.95 (s, 1H, H-5). Mass (m/z, \%): $614\left(\mathrm{M}^{+}, 14\right)$.

Anal. Calc. $\mathrm{C}_{27} \mathrm{H}_{27} \mathrm{FN}_{6} \mathrm{O}_{10}$ (614.54): C, 52.77, H, 4.43, F, 3.09, N, 13.68. Found: C, 52.75, H, 4.45, F, 3.05, N, 13.60\%.

(9c): yield $67 \%(\mathrm{~A}), 62 \%(\mathrm{~B}), 70 \%(\mathrm{C})$, m.p. $207-209^{\circ} \mathrm{C}$ (ethanol). IR (KBr) v: $1750(\mathrm{C}=\mathrm{O}) .{ }^{1} \mathrm{H}-\mathrm{NMR} \delta: 2.00,2.05,2.10,2.49\left(1 \mathrm{~d}, 3 \mathrm{~s}, 15 \mathrm{H}, \mathrm{CH}_{3}\right) ; 3.95,4.18$ $\left(2 \mathrm{~m}, 2 \mathrm{H}, \mathrm{CH}_{2}\right) ; 5.25(\mathrm{~m}, 1 \mathrm{H}, \mathrm{CH}) ; 5.30(\mathrm{~m}, 1 \mathrm{H}, \mathrm{CH}) ; 5.40(\mathrm{~m}, 1 \mathrm{H}, \mathrm{CH}) ; 5.55$ (m, 1H, CH), 7.40 (m, 2H, 2CH(Ar)); 7.90 (m , 2H, 2CH(Ar)); 8.40 (s, 1H, H-8 ); 8.66 (s, 1H, H-5). Mass (m/z, \%): $614\left(\mathrm{M}^{+}, 0.37\right)$. 
Anal. Calc. $\mathrm{C}_{27} \mathrm{H}_{27} \mathrm{FN}_{6} \mathrm{O}_{10}$ (614.54): C, 52.77, H, 4.43, F, 3.09, N, 13.68 . Found: C, 52.65, H, 4.45, F, 3.00, N, 13.70\%.

(9d): yield $65 \%(\mathrm{~A}), 63 \%(\mathrm{~B}), 68 \%(\mathrm{C})$, m.p. $271-273^{\circ} \mathrm{C}$ (ethanol). IR (KBr) v: $1750(\mathrm{C}=\mathrm{O}) .{ }^{1} \mathrm{H}-\mathrm{NMR} \delta: 2.02,2.07,2.10,2.16,2.50\left(5 \mathrm{~s}, 15 \mathrm{H}, 5 \mathrm{CH}_{3}\right) ; 4.25$, $2.33\left(2 \mathrm{~m}, 2 \mathrm{H}, \mathrm{CH}_{2}\right) ; 5.35(\mathrm{~m}, 1 \mathrm{H}, \mathrm{CH}) ; 5.87(\mathrm{~m}, 1 \mathrm{H}, \mathrm{CH}) ; 6.20(\mathrm{~m}, 1 \mathrm{H}, \mathrm{CH}$ ); $6.55(\mathrm{~m}, 1 \mathrm{H}, \mathrm{CH}), 7.40(\mathrm{~m}, 2 \mathrm{H}, 2 \mathrm{CH}(\mathrm{Ar})) ; 7.80(\mathrm{~m}, 2 \mathrm{H}, 2 \mathrm{CH}(\mathrm{Ar})) ; 8.49(\mathrm{~s}$, 1H, H-8); 8.67 (s, $1 \mathrm{H}, \mathrm{H}-5)$. Mass (m/z, \%): $614\left(\mathrm{M}^{+}, 0.60\right)$.

Anal. Calc. $\mathrm{C}_{27} \mathrm{H}_{27} \mathrm{FN}_{6} \mathrm{O}_{10}$ (614.54): C, 52.77, H, 4.43, F, 3.09, N, 13.68. Found: C, 52.80, H, 4.40, F, 3.10, N, 13.65\%.

1-[7-(4-Fluorophenyl)-7H-[1,2,4]triazolo[3,4-i]-purin-3-yl]butane-1,2, 3,4-tetraol (10a) and 1-[7-(4-fluorophenyl)-7H-[1,2,4]triazolo [3,4-i]- purin-3-yl]pentane-1,2, 3,4,5-pentols (10b-d)

General procedure

A suspension of the appropriate (7a-d) (0.004 mol.) in water ( $3 \mathrm{ml})$ was drop wisely treated with bromine $(0.003 \mathrm{~mol})$ in water $(3 \mathrm{ml})$ while stirring. The reaction mixture was kept overnight at room temperature (ca. $25^{\circ} \mathrm{C}$ ). The solvent was evaporated to dryness under reduced pressure, the obtained reduced was crystallized from water/ethanol to give (10a-d).

(10a): yield $63 \%$, m.p. $318-320^{\circ} \mathrm{C}$ (aq. ethanol). IR ( $\left.\mathrm{KBr}\right)$ v: 3462,3420 $(\mathrm{NH})$. Mass $(\mathrm{m} / \mathrm{z}, \%): 374\left(\mathrm{M}^{+}, 0.10\right)$.

Anal. Calc. $\mathrm{C}_{16} \mathrm{H}_{15} \mathrm{FN}_{6} \mathrm{O}_{4}$ (374.33): C, 51.34, H, 4.04, F, 5.08, N, 22.45 . Found: C, 51.35, H, 4.00, F, 5.05, N, 22.40\%.

(10b): yield 70\%, m.p. $300-302^{\circ} \mathrm{C}$ (aq. ethanol). IR (KBr) v: 3346, 3330 $(\mathrm{NH}){ }^{1} \mathrm{H}-\mathrm{NMR} \delta: 4.04(\mathrm{~m}, 1 \mathrm{H}, \mathrm{CH}) ; 4.12(\mathrm{~m}, 1 \mathrm{H}, \mathrm{CH}) ; 5.06(\mathrm{~m}, 1 \mathrm{H}, \mathrm{CH}) ; 5.08$ $(\mathrm{m}, 1 \mathrm{H}, \mathrm{CH}) ; 6.11(\mathrm{~b}, 1 \mathrm{H}, \mathrm{OH}) ; 6.47(\mathrm{~b}, 1 \mathrm{H}, \mathrm{OH}) ; 7.51(\mathrm{~b}, 1 \mathrm{H}, \mathrm{OH}) ; 7.53(\mathrm{~m}, 2 \mathrm{H}$, $2 \mathrm{CH}(\mathrm{Ar})) ; 7.88(\mathrm{~d}, 1 \mathrm{H}, \mathrm{H}-3) ; 7.90(\mathrm{~m}, 1 \mathrm{H}, \mathrm{CH}(\mathrm{Ar})) ; 8.81$ (s, 1H, H-8); 9.48 (s,1H,H-5).

Anal. Calc. $\mathrm{C}_{17} \mathrm{H}_{17} \mathrm{FN}_{6} \mathrm{O}_{5}$ (404.35): C, 50.50, H, 4.24, F, 4.70, N, 20.78. Found: C, 50.55, H, 4.20, F, 4.75, N, 20.73\%.

(10c): yield $65 \%$, m.p. $315-317^{\circ} \mathrm{C}$ (aq. ethanol). IR (KBr) v: 3450,3356 $(\mathrm{NH})$. Mass $(\mathrm{m} / \mathrm{z}, \%): 404\left(\mathrm{M}^{+}, 0.15\right)$.

Anal. Calc. $\mathrm{C}_{17} \mathrm{H}_{17} \mathrm{FN}_{6} \mathrm{O}_{5}$ (404.35): C, 50.50, H, 4.24, F, 4.70, N, 20.78. Found: C, 50.60, H, 4.22, F, 4.77, N, 20.75\%.

(10d): yield $63 \%$, m.p. $314-316^{\circ} \mathrm{C}$ (aq. ethanol). IR (KBr) v: 3418,3350 $(\mathrm{NH})$.

Anal. Calc. $\mathrm{C}_{17} \mathrm{H}_{17} \mathrm{FN}_{6} \mathrm{O}_{5}$ (404.35): C, 50.50, H, 4.24, F, 4.70, N, 20.78. Found: C, 50.40, H, 4.25, F, 4.65, N, 20.75\%.

Acknowledgement:Thanks are due to the National Research Centre for the financial support. 
References

1. (a) Alberts, B., Bray, D., Lewis, J., Raff, M., Roberts, K. and Watson, J.D., Molecular Biology of the Cell, $3^{\text {rd }}$ ed.; Garland: New York, 736 (1994). (b) Gao, Z., Duhl, D.D. and Harrison, S.D., Ann. Rep. Med. Chem. 38, 193-201 (2003).

2. Legraverend, M., Ludwig, O., Bisagni, E., Leclerc, S. and Meijer, L., Bioorg. Med.Chem. Lett. 8, 793-798(1998).

3. Furet, P., Zimmermann, J., Capraro, H.G., Meyer, T. and Imbach, P., $J$. Comput.-Aided Mol. Des. 14, 403-409(2000).

4. Legraverend, M., Tunnah, P., Noble, M., Ducrot, P., Ludwig, O., Grierson, D.S., Leost, M., Meijer, L. and Endicott, J., J. Med. Chem. 43, 1282-1292 (2000).

5. Sielecki, T.M., Boylan, J.F., Benfield, P.A. and Trainor, G.L., J. Med. Chem. 43, $1-18(2000)$

6. Ducrot, P., Legraverend, M. and Grierson, D.S., J. Med. Chem. 43, 4098-4108 (2000).

7. Wanner, M.J., Von Frijtag Drabbe, J.K., Ijzerman, A.P. and Koomen, G., $J$. Bioorg. Med. Chem. Lett. 10, 2141-2144(2000).

8. Abiru, T., Miyashita, T., Watanabe, Y., Yamaguchi, T., Machida, H. and Matsuda, A., J. Med. Chem. 35, 2253-2260 (1992).

9. Harada, H., Asano, O., Hoshino, Y., Yoshikawa, S., Matsukura, M., Kabasawa, Y., Niijima, J., Kotake, Y., Watanabe, N., Kawata, T., Inoue, T., Horizoe, T., Yasuda, N., Minami, H., Nagata, K., Murakami, M., Nagaoka, J., Kobayashi, S., Tanaka, I. and Abe, S., J. Med. Chem. 44, 170-179 (2001).

10. Dhainaut, A., Regnier, G., Tizot, A., Pierre, A., Leonce, S., Guilbaud, N., Kraus-Berthier, L. and Atassi, G., J. Med. Chem. 39, 4099-4108(1996).

11. Gao, H. and Mitra, A.K., Synthesis, 329-351(2000).

12. Bonnet, P.A. and Robins, R.K., J. Med. Chem. 36, 635-653(1993).

13. Capek, P., Pohl, R. and Hocek, M., J.Org. Chem. 70, 8001-8008(2005).

14. (a) De Clercq, E., "Design of Anti-AIDSDrugs",De Clercq, E., (Ed.) Elsevier: Amesterdam, pp. 1-95 (1990) (b) Huryn, D.M., and Okabe, M., Chem. Review, 92,1745- 1768(1992). (c) Perigaud, C., Gosselin, G. and Imbach,J.-L., Nucleosides \& Nucleotides, 11, 903-945(1992).

15. (a) Hacksell, U. and. Daves, G.D., Jr. Prog. Med. Chem. 22,1-65(1985). (b) Postema, M.H.D., Tetrahedron, 48, 8545-8599(1992).(c) Watanabe, K.A., "Chemistry of Nucleosides and Nucleotides";Towensend, L.B. (Ed); Plenum press: New York, N.Y., 3, 421. (1994) (d) Jaramillo, C. and Knapp, S., Synthesis, 
20(1994).(e) Togo, H., He, W., Waki, Y. and Yokoyama, M., Synnlett, 700717(1998).

16. (a) Shaban, M.A.E. and Nasr, A.Z., Adv. Heterocycl. Chem. 68, 223-432(1997). (b) Shaban, M.A.E., Adv. Heterocycl Chem. 70, 163 (1998).

17. Clark, J.L., Mason, J., Hollecker, C L., Stuyver, L.J., Tharnish, P.M., McBrayer, T.R., Otto, M.J., Furman, P.A., Schinazi, R.F. and Watanabe, K.A., Bioorganic \& Medicinal Chemistry Letters, 16, 1712-1715 (2006).

18. Mamat, C., Hein, M. and Miethchen, R., Carbohydr. Res. 341, 1758-1763 (2006).

19. El-Bayouki, Kh.A.M., Basyouni, W.M. and Tohamy, W.M., Egypt. J. Chem. 53, 803-820 (2010).

20. (a) Gürsoy, A. and Terzioğlu, N., Turk J. Chem. 29, 247-254 (2005). (b) Karali, N., Gürsoy, A., Terzioğlu, N., Ozkirimli, S., Özer, H. and Ekinci, A.C., Arch. Pharm. Pharm. Med. Chem. 331, 254-258 (1998).

21. Shaban, M.A.E., Taha, M.A.M., Nasr, A.Z. and Morgaan, A.E. A., Pharmazie, 50, $784-788$ (1995).

(Received 23/4/2013; accepted 11/6/2013) 


\section{تثييد مشتقات جديدة من البيورينات و[1،1،2]تترايازولو[3،3] بيورينات}

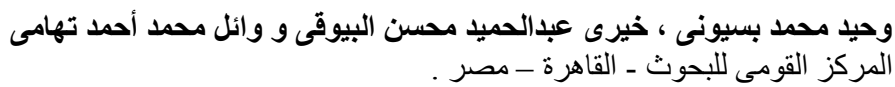

تم فى هذا البحث الحصول على مشتقات البيورين الجديدة وذلك من خلال مفاعلة

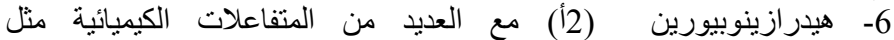

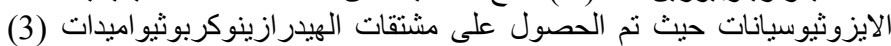

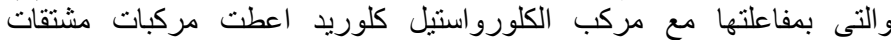

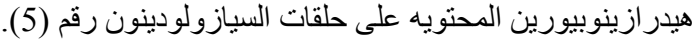

وقد تم ايضا مفاعلة المركب (2أ) مع العديد من السكريات الاحادية حيث تم

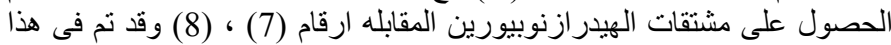

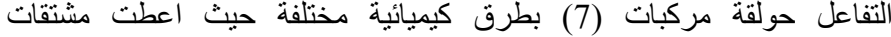
التر ايازولوبيورين المقابله ارقام (9) ، (10) (10). 